\title{
Method of identification of parameters of closed planetary train based on exploratory design
}

\author{
Ildar Kutlubaev ${ }^{1}$, Elena Matcko ${ }^{1}$, and Olga Panfilova ${ }^{1, *}$ \\ ${ }^{1}$ Department of Mining Machines and Transport-Technological Complexes, Nosov Magnitogorsk \\ State Technical University, 38 Lenina prospect, Magnitogorsk 455000, Russia
}

\begin{abstract}
Practicability of usage of gears with closed planetary trains in high-powered drives having weight and dimensions limitations is represented. Method that makes it possible to perform evaluation of massdimensional characteristics of planetary double-flow gears at the exploratory design stage is proposed. The developed approach makes it possible to determine basic geometric parameters of gear-tooth system: diameters and width of tooth-wheels reasonably. Besides the obligatory fulfillment of contact tooth strength conditions, providing of the required transmission ratio and condition of coincidence of axes of the gear input and output shafts, the condition of its minimum weight and dimensions is taken into account either. The last requirement is achieved by determination of conditional factor that is square of kinematic scheme contour expressed through projected parameters. The minimum value of contour square corresponds to the best combination of diameters and width values of tooth wheels if all functional limitations are maintained. Then the need of arbitrary choice of any of projected parameters and further verification of the choice made at the closing designing stage is excepted. The proposed approach makes it possible to evaluate the efficiency of chosen gear structural scheme at the initial design stage without design study of workpieces and units.
\end{abstract}

Efficiency of gears of technological and transport machines is provided by means of upgrading of efficiency coefficient and power-weight ratio. In this case the general trend for all types of drives is the increase of the engine speed. The rotational rate of the modern engines especially of small powered drives reaches $600-1000 \mathrm{~s}^{-1}$ [1]. Hydraulic oil motors as a rule have speed from 15 to $260 \mathrm{~s}^{-1}$ [2].

This factor determined the need of increasing of the gears transmission ratio. Harmonic drives are optimized with respect to transmission ratio/dimensions balance. Planetary gears slightly come short of this criterion. Thus harmonic drives have limitation of the value of transferred rotational torque. Harmonic Drive LLC, production leader of harmonic drives, produces items with maximal output torque up to $3419 \mathrm{~N} \cdot \mathrm{m}$ [3]. However, the main range has the torque up to $2630 \mathrm{~N} \cdot \mathrm{m}$.

Besides that, harmonic drives are characterized by instability of efficiency coefficient in case of continuous work.

\footnotetext{
*Corresponding author: halikova@inbox.ru
} 
The usage of planetary gears is more preferable in high-powered drives. Its constructions are continually-improving, new variations of toothing constructions are developed $[4,5]$. Known factors mainly connected with accuracy of manufacturing and influence of wear problem on efficiency coefficient [6] restrain its wide-spread occurrence.

Increasing of power transferred by a gear can be obtained by means of development of constructions that realize traditional construction schemes, in particular through providing of higher precision of power distribution through parallel flows [7, 8].

Capability to obtain high transmission ratio and rotational torque simultaneously is reached by usage of double-flow schemes with nonparallel flows of transferred power [9, $10,11]$.

Development of working project includes stage of choice of structural scheme and calculation of main parameters of kinematic scheme. In this case performance evaluation of chosen gear structural scheme is performed according to the results of finished working project. Particularly according to the power-weight ratio coefficient

$$
N_{p w}=\frac{T_{\text {out }} \cdot \omega_{\text {out }}}{m},
$$

where $T_{\text {out }}$ - torque at the output shaft;

$\Omega_{\text {out }}-$ angular velocity of output shaft;

$m$ - gear weight.

However, the gear weight is determined at the conclusion of project. In this regard preliminary analysis of possible variations of structural schemes according to indirect index of power-weight ratio becomes essential. In this regard it is reasonable to have a criterion that makes it possible to evaluate the accepted working kinematic scheme at the initial design stage.

The expression (1) contains parameter $m$, its value is determined in a great measure by the volume of the gear internal space which is full of wheels. In this regard before engineering development the evaluation of the gear weight corresponding to the accepted scheme is possible to perform according to the conditional index that is square of kinematic scheme contour $K_{s c}$. Its value depends on the projected parameters such as reference circle diameter of wheels $d_{i}$ and width $b_{j}$ (figure 1). In this case index $K_{s c}$ can be a criterion of optimality determined by the set of projected parameters

$$
K_{s c}=b_{1} \cdot d_{3}+b_{2} \cdot d_{6} .
$$

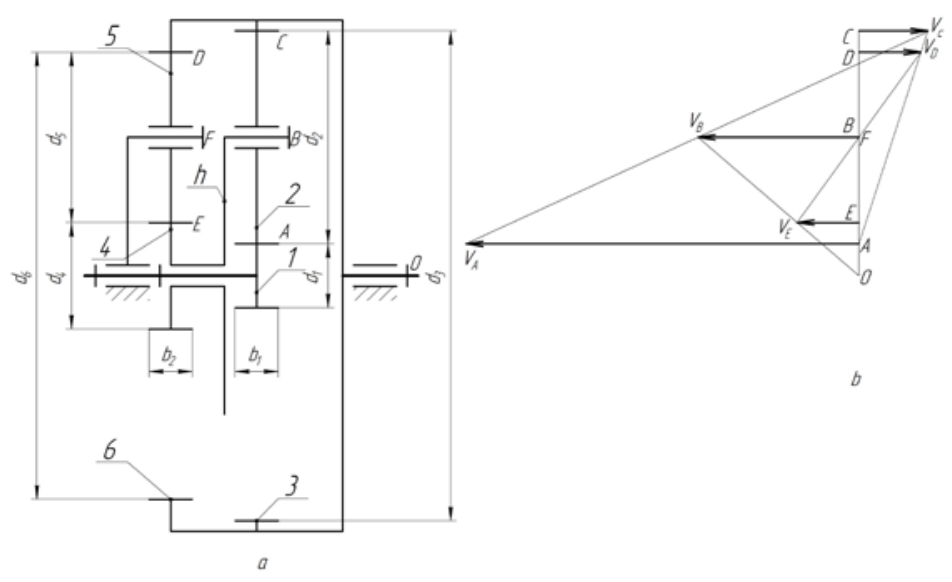

Fig. 1. Double-flow planetary gear with nonparallel flows: $a$ - kinematic scheme, $b$ - velocity plan 
In respect to the scheme of double-flow planetary gear the number of parameters to be determined equals eight: $d_{i}(i=1 \ldots 6), b_{j}(j=1 \ldots 2)$.

Parameters that determine the gear scheme are connected with each other by obligatory correlations that are functional limitations placed on criterion $K_{s c}$ :

- maintenance of the required transmission ratio $u$ [9]

$$
u=2 \cdot \frac{d_{6} \cdot\left(d_{1}+d_{2}\right)}{d_{4} \cdot d_{1}}+\frac{d_{3}}{d_{1}}
$$

- fulfillment of conditions of tooth strength [12]

$$
\begin{aligned}
& d_{1}=K_{d} \cdot \sqrt[3]{\frac{T_{2} \cdot d_{1} \cdot\left(d_{1}+d_{2}\right)}{2 \cdot \psi_{b d 1} \cdot d_{2}^{2} \cdot\left[\sigma_{H}\right]^{2}}} ; \\
& d_{4}=K_{d} \cdot \sqrt[3]{\frac{T_{5} \cdot d_{4} \cdot\left(d_{4}+d_{5}\right)}{2 \cdot \psi_{b d 2} \cdot d_{5}{ }^{2} \cdot\left[\sigma_{H}\right]^{2}}},
\end{aligned}
$$

where $K_{d}=840$ for spur gear pairs; $K_{d}=780$ for helical-gear set;

$K_{C}$ - coefficient of irregularity in the distribution of load between satellite gears; $K_{C}=$ $1,1 \ldots 1,2$

$T_{2}$ and $T_{5}$ - rotational torques on annular rings 2 and 5;

$K_{1}$ and $K_{2}$ - number of satellite gears of the first and second flows of power transmission correspondingly;

$\psi_{b d 1}$ and $\psi_{b d 2}$ - coefficients of toothing width:

$$
\psi_{b d 1}=\frac{b_{1}}{d_{1}} ; \quad \psi_{b d 2}=\frac{b_{2}}{d_{4}} ;
$$

- condition of coincidence of axes

$$
d_{3}=d_{1}+2 \cdot d_{2} ; \quad d_{6}=d_{4}+2 \cdot d_{5} .
$$

In the equations (4) and (5) the calculation is performed through rotational torques in nonparallel flows $T_{2}$ and $T_{5}$. Distribution of pull-in torque $T_{1}$ to flows is determined by geometric parameters based on force analysis (figure 2).

The width of rim of each wheel depends on applied loads determined during force analysis.

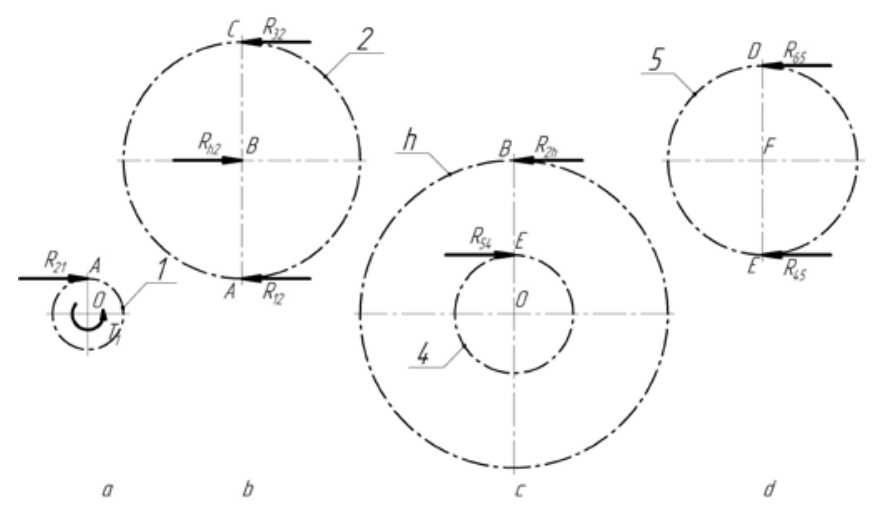

Fig. 2. Loads in toothing of double-flow planetary gear with nonparallel flows 
The load in toothing between links 1 and 2 that is in point $A$ (figure 2, $a$ ) is determined by the equation of equality of torques operate on annular ring 1 (about an axis $O$ )

$$
R_{21}=\frac{K_{C} \cdot 2 \cdot T_{1}}{K_{1} \cdot d_{1}}
$$

where $K_{C}$ - coefficient of irregularity in the distribution of load between satellite gears;

$K_{1}$ - the number of satellite gears in the first flow of power transmission.

Forces applied to link 2 on the part of link 3 in point $C$ and link $h(4)$ in point B are determined by the condition of equality of torques applied to satellite gear 2 about points $B$ and $C$ (figure $2, b$ )

$$
\begin{gathered}
R_{32}=-R_{12}-R_{21}=\frac{K_{C} \cdot 2 \cdot T_{1}}{K_{1} \cdot d_{1}} \\
R_{h 2}=-\left(R_{12}+R_{32}\right)=-\frac{K_{C} \cdot 4 \cdot T_{1}}{K_{1} \cdot d_{1}}
\end{gathered}
$$

The loading in toothing (point E) between links 4 and 5 is determined by condition of equality of torques applied to link $h(4)$ (figure 2,c)

$$
R_{54}=\frac{R_{2 h} \cdot K_{C} \cdot K_{1} \cdot\left(d_{1}+d_{2}\right)}{K_{2} \cdot d_{4}}=\frac{4 \cdot T_{1} \cdot K_{C} \cdot\left(d_{1}+d_{2}\right)}{K_{2} \cdot d_{1} \cdot d_{4}} .
$$

Then the load in point $\mathrm{D}$ is determined by the condition of equality of torques applied to satellite gear 5 about axis $F$ (figure 2,d),

$$
R_{65}=R_{45}=\frac{4 \cdot T_{1} \cdot K_{C} \cdot\left(d_{1}+d_{2}\right)}{K_{2} \cdot d_{1} \cdot d_{4}} .
$$

As a result, loads applied to each of fixedly connected toothed rims 3 and 6 (forces in points $\mathrm{C}$ and $\mathrm{D}$ correspondingly) are known during consideration of condition of equilibrium of output link 3(6) at the concluding stage of force analysis. This makes it possible to determine the rotational torque at the gear output shaft.

The results of the accomplishment of force analysis in the presented consequence are expressions of rotational torques:

$$
\begin{gathered}
T_{2}=\frac{K_{C} \cdot 2 \cdot T_{1} \cdot d_{2}}{K_{1} \cdot d_{1}} ; \\
T_{3}=\frac{T_{1} \cdot d_{3}}{d_{1}} ; \\
T_{4}=\frac{2 \cdot T_{1} \cdot\left(d_{1}+d_{2}\right)}{d_{1}} ; \\
T_{5}=\frac{K_{C} \cdot 2 \cdot T_{4} \cdot d_{5}}{K_{2} \cdot d_{4}}=\frac{K_{C} \cdot 4 \cdot T_{1} \cdot\left(d_{1}+d_{2}\right) \cdot d_{5}}{K_{2} \cdot d_{1} \cdot d_{4}} ;
\end{gathered}
$$




$$
T_{6}=\frac{2 \cdot T_{1} \cdot\left(d_{1}+d_{2}\right) \cdot d_{6}}{d_{1} \cdot d_{4}} .
$$

The correlation of rotational torques transferred by nonparallel power flows expressed by geometric parameters of a mechanism

$$
\frac{T_{3}}{T_{6}}=\frac{d_{3} \cdot d_{4}}{2 \cdot\left(d_{1}+d_{2}\right) \cdot d_{6}}
$$

The width of wheels is expressed during the solution of equations (6) and (7) including (4) and (5) correspondingly

$$
\begin{aligned}
& b_{1}=K_{d} \cdot \sqrt[3]{\frac{\psi_{b d 1}^{2} \cdot T_{2} \cdot d_{1} \cdot\left(d_{1}+d_{2}\right)}{2 \cdot d_{2}^{2} \cdot\left[\sigma_{H}\right]^{2}}} \\
& b_{2}=K_{d} \cdot \sqrt[3]{\frac{\psi_{b d 2}^{2} \cdot T_{5} \cdot d_{4} \cdot\left(d_{4}+d_{5}\right)}{2 \cdot d_{5}^{2} \cdot\left[\sigma_{H}\right]^{2}}}
\end{aligned}
$$

The insertion of correspondences (7), (8), (9) into expression (2) makes it possible to introduce functional limitations into criterion of optimality $K_{s c}$. As a result, one functional limitation in the form of equation (3) is applied to criterion $K_{s c}$. In some cases, to simplify the procedure of a function extremum seeking it is reasonable to change the condition of equation to inequation

$$
2 \cdot \frac{d_{6} \cdot\left(d_{1}+d_{2}\right)}{d_{4} \cdot d_{1}}+\frac{d_{3}}{d_{1}}-\Delta u \geq 0,
$$

where $\Delta u$ - acceptable deviation of transmission ratio $u$.

Projected parameters determined by the condition of minimum criterion of optimality and considering functional limitation correspond to better mass-dimensional characteristics of a gear. In this case the obligatory limitations to contact tooth strength, required transmission ratio of a gear and coincidence of axes of its input and output shafts are performed.

This method can be used either for exploratory design of double planetary gears. In this case modules of toothing for each step should be included in the number of projected parameters.

\section{Conclusion}

The decision to use planetary gears with two nonparallel flows of transferred power especially with small size and weight requirements in high-powered gears is rational.

The issue of rational and reasonable choice of main geometric parameters of toothing system is decided even on an exploratory design stage on the basis of evaluation of conditional factor that is square of kinematic scheme contour. This makes it possible to provide the best variant of combination of parameters according to the power-weight ratio coefficient even at the initial design stage. 
The developed approach minimizes the scope of calculations needed for evaluation of efficiency of the gear kinematic scheme and makes it possible to perform it before the details and units design.

The proposed method can be applied to gears of different structural schemes as it presents the generals terms of functioning and compactability requirements.

\section{References}

1. MaxonMotor. Driven by Precision [electronic resource] https://www.maxonmotor.com (reference date 01.04.2018)

2. Danfoss - EngineeringTomorrow, Today [electronic resource] https://www.danfoss.com (reference date 01.04.2018)

3. Harmonic Drive [electronic resource] https://www.harmonicdrive.net (reference date 01.04.2018)

4. V.V. Morozov, Modern problems of science and education 6 [electronic resource] http://science-education.ru/ru/article/view?id=4918 (reference date 24.03.2018) (2011)

5. D.S. Blinov, M.I. Morozov, Proc. of Higher Educational Institutions. Machine Building 3, 62-72 (2013)

6. D.S. Blinov, V.O. Vasilyuk, Machines and Plants: design and exploiting 3, 23-27 (2016)

7. A.V. Romanov, Bulletin of Kalashnikov ISTU 1 (69), 22-25 (2016)

8. Yu.V. Turygin, Yu.V. Zubkova, R. Gartyanski, Mechatronics, automation, control 7, 35-41 (2014)

9. I.M. Kutlubaev, E.Yu. Matcko, O.R. Panfilova, MATEC Web of Conferences 129 06023 DOI: 10.1051/matecconf/201712906023 (2017)

10. J. Drewniak, P. Garlicka, A. Kolber, Scientific Journal of Silesian University of Technology. Series Transport 91, 5-17 (2016)

11. A.L. Kapelevich, V.M. Ananiev, Proc. of the ASME IDETC/CIE 8, 27-133 (2011)

12. D.N. Reshetov, A.P. Gusenkov, Yu.N. Drozdov et al. under the general editorship of D.N. Reshetov. Mashinostroenie. Enciklopediya. Detali mashin. Konstrukcionnaya prochnost'. Trenie, iznos, smazka (Machinery. Encyclopaedia. Machinery parts. Structural strength. Friction, wear, oiling.) IV-1, 864 (1995) 